\title{
First-line Helicobacter pylori eradication therapies in countries with high and low clarithromycin resistance: a systematic review and network meta-analysis
}

\author{
Yee Hui Yeo, ${ }^{1,2}$ Sz-luan Shiu, ${ }^{1}$ Hsiu J Ho, ${ }^{1}$ Biyao Zou, ${ }^{3}$ Jaw-Town Lin, ${ }^{4,5}$ \\ Ming-Shiang $\mathrm{Wu}^{6}{ }^{6}$ Jyh-Ming Liou, ${ }^{6}$ Chun-Ying $\mathrm{Wu}_{1}{ }^{1,6,8,9,10}$ \\ Taiwan Gastrointestinal Disease and Helicobacter Consortium
}

\begin{abstract}
- Additional material is published online only. To view please visit the journal online (http://dx.doi.org/10.1136/ gutjnl-2016-311868)
\end{abstract}

For numbered affiliations see end of article.

Correspondence to Professor Chun-Ying Wu, Division of Gastroenterology and Hepatology, Taichung Veterans General Hospital 1650, Sec. 4, Taiwan Boulevard, Taichung 40705, Taiwan; chun@vghtc.gov.tw

Received 15 March 2016 Revised 1 September 2016 Accepted 2 September 2016 Published Online First 26 September 2016

\section{ABSTRACT \\ Objective To determine the optimal regimen of} different first-line Helicobacter pylori eradication therapies according to the clarithromycin resistance rate. Design Electronic search for articles published between January 2005 and April 2016. Randomised, controlled trials that reported the effectiveness of first-line eradication therapies in treatment-naïve adults were included. Two independent reviewers performed articles screening and data extraction. Network and traditional meta-analyses were conducted using the random effect model. Subgroup analyses were performed to determine the ranking of regimens in countries with high (>15\%) and low $(<15 \%)$ clarithromycin resistance. Data including adverse events and therapeutic cure rate were also extracted and analysed.

Results 117 trials (totally 32852 patients) for 17 H. pylori eradication regimens were eligible for inclusion. Compared with 7-day clarithromycin-based triple therapy, sequential therapy (ST) for 14 days had the highest effectiveness ( $\mathrm{OR}=3.74,95 \% \mathrm{Crl} 2.37$ to 5.96$)$. ST-14 $(\mathrm{OR}=6.53,95 \% \mathrm{Crl} 3.23$ to 13.63$)$ and hybrid therapy (HY) for 10 days or more $(\mathrm{OR}=2.85,95 \% \mathrm{Crl} 1.58$ to 5.37) represented the most effective regimen in areas with high and low clarithromycin resistance, respectively. The effectiveness of standard triple therapy was below therapeutic eradication rate in most of the countries. Longer duration was associated with higher eradication rate, but with a higher risk of events that lead to discontinuation.

Conclusions ST and HY appeared to be the most effective therapies in countries with high and low clarithromycin resistance, respectively. The clinical decision for optimal regimen can be supported by referring to the rank ordering of relative efficacies stratified by local eradication rates, antibiotic resistance and safety profile.

Trial registration number CRD42015025445.

\section{INTRODUCTION}

CrossMark

To cite: Yeo YH, Shiu S-I,

Ho HJ, et al. Gut

2017:67:20-27.
Helicobacter pylori infection is present in more than half of the population worldwide. ${ }^{1}$ It is causally associated with the pathogenesis of peptic ulcer disease, active gastroduodenal ulcer bleeding, gastric mucosa-associated lymphoid tissue lymphoma, and is accountable for more than

\section{Significance of this study}

What is already known on this subject?

- Eradication rate of conventional triple therapy has declined progressively worldwide, particularly in clarithromycin-based triple therapy.

- Several regimens such as concomitant therapy, sequential therapy and hybrid therapy have been proposed to improve the eradication rate.

What are the new findings?

- Overall, sequential therapy for 14 days presented the highest eradication rates.

- Sequential therapy and hybrid therapy appeared to be the most effective therapies in areas with high and low clarithromycin resistance, respectively.

- Longer duration was associated with increased effectiveness, and also with higher risk of events that lead to discontinuation.

How might it impact on clinical practice in the foreseeable future?

- Our report presented evidence-based hierarchy for the effectiveness of 17 eradication regimens according to clarithromycin resistance rate.

- Our report provides significant insights into the compositional effects of regimen type and duration, adverse events and therapeutic cure rate to facilitate physician's decision-making process respecting optimal regimen selection and treatment duration.

three-quarter of all gastric cancer cases. ${ }^{2} 3$ The eradication of $H$. pylori has been recommended in patients with long-term use of non-steroidal antiinflammatory drugs (NSAIDs), antiplatelet agent or low-dose aspirin, as well as for patients with unexplained iron-deficiency anaemia or idiopathic thrombocytopenic purpura to improve the patients' prognosis. 4

Standard triple therapy (TT), which consists of proton pump inhibitor (PPI), amoxicillin, 
clarithromycin or metronidazole, was developed in the 1990s and recommended as the first-line eradication therapy due to favourable eradication rate initially. In recent years, mounting evidence has indicated progressively declining eradication rate for $\mathrm{TT}^{5-7}$ Alternative combination regimens have been proposed, including bismuth-containing quadruple therapy (BQT), non-bismuth quadruple therapy (also called concomitant therapy, CT), sequential therapy (ST), hybrid therapy (HY) and quinolone-based TT. Their efficiencies have been tested in different countries, including countries with high or low clarithromycin resistance. However, the optimal regimen for $H$. pylori eradication remains elusive. Moreover, there is little evidence on the therapeutic eradication rate (higher than $80 \%{ }^{4}{ }^{8}$ ) of different regimens in different countries.

In search of the optimal eradication therapy, systematic reviews have focused on the relative effectiveness of standard TT compared with the newer regimens. However, comparative studies of new regimens are still limited. There are two major obstacles to conducting traditional meta-analyses for comparisons of multiple regimens. The first is the difficulty in applying conventional pairwise meta-analyses to the integration and systematic comparison of more than two eradication regimens. The second is that not all of the eradication regimens have been compared head-to-head via randomised controlled trial (RCT). Network meta-analysis can be used to investigate several treatment comparators simultaneously with direct and indirect comparisons. ${ }^{9-11} \mathrm{Li}$ and colleagues recently reported a network meta-analysis to explore the most efficacious therapy for eradication of $\mathrm{H}$. pylori. ${ }^{12}$ However, the inclusion of early papers, incomplete inclusion of trials and the comparison of nonmainstream regimens such as probiotics and $\mathrm{H} 2$ blocker containing therapies may result in higher heterogeneity and inconsistency.

In the present study, we conduct network meta-analysis to compare the relative efficacies and tolerabilities of 17 key regimens in the eradication of $H$. pylori to determine the most favourable regimens for treatment-naïve patients. To enhance the clinical significance, we performed subgroup analysis to determine the rank ordering of regimens in countries with high $(>15 \%)$ and low $(<15 \%)$ clarithromycin resistance. We also conducted a systematic review to explore the severe adverse event rates and the weighted eradication rate of different therapies in each study country.

\section{MATERIALS AND METHODS}

\section{Data sources and searches}

We searched PubMed and Cochrane Central Register of Controlled Trials to identify relevant trials without language restriction. Detailed search strategies are described in the online supplementary appendix 1 . We also scanned the reference lists of reviews and included studies for additional trials. Antibiotic resistance may rise incrementally and impose bias. Similar to a recent consensus, we restricted our search to full-text articles published from 2005 to 2016 April. $^{13}$ The preplanned protocol of our network meta-analysis is documented online (PROSPERO registry-CRD42015025445). The study protocol was approved by the institutional review boards of Taichung Veterans General Hospital.

\section{Study selection}

We included RCTs for the evaluation of $H$. pylori eradication rate in treatment-naïve adults (aged 18 years or older) with a diagnosis of $\mathrm{H}$. pylori infection according to one or more of the standard detection methods (13C-urea breath test, histological examination, bacterial culture, rapid urea test or stool antigen detection). Eligible reports were those that compared two or more of the 17 studied regimens. H. pylori eradication rate was determined by intention to treat analysis at least 4 weeks after completion of eradication regimen. Studies of paediatric patients or patients with severe comorbidities were excluded, as were those that included probiotics, $\mathrm{H} 2$ blockers and herbs.

Two investigators checked titles, abstracts and full texts independently to clarify the eligibility of each article. All discrepancies were discussed and resolved in consultation with a third investigator. Non-English articles were translated when needed. We attempted to contact investigators to acquire full text or to clarify and obtain study results. Finally, articles without full text available from the authors and abstracts from conferences were excluded.

\section{Data extraction and quality assessment}

The following variables were extracted by two investigators independently: participant demographic characteristics, methods for detecting $H$. pylori infection, details of regimens, the washout period of antibiotics, PPIs, aspirin, use of NSAIDs, eradication rates and methods and time points for detecting H. pylori eradication. We also derived compliance rates, severe adverse events and events that were responsible for drugs discontinuation from included studies.

We grouped the 17 included regimens according to clinical similarity: clarithromycin-based triple therapy for 7 days (TT-C-7), clarithromycin-based triple therapy for 10 days (TT-C-10), clarithromycin-based triple therapy for 14 days (TT-C-14), quinolone-based triple therapy for 7 days (TT-Q-7), quinolone-based triple therapy for more than or equal to 10 days (TT-Q $\geq 10)$, metronidazole-based triple therapy for 7 days (TT-M-7), metronidazole-based triple therapy for more than or equal to 10 days (TT-M $\geq 10$ ), triple therapy with two major drugs (PPI+quinolone+clarithromycin or quinolone +metronidazole) for 7 days (TT-D-7), bismuth-containing quadruple therapy (bismuth + PPI+tetracycline + metronidazole) for less than or equal to 10 days $(\mathrm{BQT} \leq 10)$, bismuth-containing quadruple therapy for 14 days (BQT-14), modified bismuthcontaining quadruple therapy (bismuth+PPI+any two of amoxicillin/tetracycline/metronidazole/clarithromycin) for less than or equal to 10 days (mBQT $\leq 10)$, modified bismuthcontaining quadruple therapy for 14 days (mBQT-14), concomitant therapy for less than or equal to 7 days $(\mathrm{CT} \leq 7)$, concomitant therapy for more than or equal to 10 days $(C T \geq 10)$, sequential therapy for less than or equal to 10 days $(\mathrm{ST} \leq 10)$, sequential therapy for 14 days (ST-14) and hybrid therapy for more than or equal to 10 days $(\mathrm{HY} \geq 10)$.

We explored the comparison network of relative estimated effects by drawing geometry of 17 regimens. In the network of regimens, each regimen is presented as a node. The lines between any two nodes represent studies comparing two linked treatments.

Two authors independently evaluated the risk of bias of all studies based on the Cochrane Handbook for Systematic Reviews of Interventions, ${ }^{14}$ with disagreements resolved through consensus. Our report was conducted based on the PRISMA extension statement for reporting of systematic reviews incorporating network meta-analyses of healthcare interventions (see online supplementary table S5). ${ }^{15}$

\section{Data synthesis and analysis}

First, we conducted traditional pairwise meta-analyses with random effects models for all regimens compared within the 
included studies using $\mathrm{R}$ software. On DerSimonian and Laird random effects models, we calculated the pooled estimates of ORs and 95\% CIs of direct comparisons between any two of the studied regimens. ${ }^{16}$

Heterogeneity of treatment effects among included studies was examined using the Cochrane $\mathrm{Q}$ test and $\mathrm{I}^{2}$ statistic. $\mathrm{I}^{2}$ of 25\%, 50\% and 75\% represent low, moderate and high heterogeneity. ${ }^{17}$ We checked for publication bias using Egger's regression asymmetry test. For Cochrane Q test and Egger's test, a two-sided $\mathrm{p}$ value of $<0.05$ was regarded as significant. We also performed netfunnel plot to examine the publication bias. ${ }^{18}$

Second, we conducted network meta-analysis in a Bayesian framework. $^{9-11}$ WinBUGS software V.1.4.3 (MRC Biostatistics Unit, Cambridge, UK) was used for estimation of effect sizes with non-informative priors. We adopted random effects models due to the nature of network meta-analysis, which comprises tolerability to between-study heterogeneity and within-study variability. Posterior distributions of model parameters were assessed based on three sets of initial values and 300000 iterations (100 000 per chain). For each chain, we discarded the first 25000 estimates as the burn-in period. A thinning interval of 10 was used to fit the model. Convergence of iterations was conducted by adopting the Gelman-Rubin-Brooks statistic. Lack of autocorrelation was confirmed for all iterations. We derived the median of posterior distributions and 95\% credible interval (CrI) $(2.5 \%-97.5 \%$ percentile) to determine the point estimate and distribution of treatment effect.

The assumption of transitivity implicates the distribution of effect modifiers is the same in all pairwise comparisons. The potential effect modifiers in our study include age, smoking status and drug adherence. Violation of transitivity would be suggested if there was inconsistency between direct and indirect results. ${ }^{19}$ Inconsistency is defined as the difference between direct and indirect evidence for each linked comparison in the network. We evaluated the differences by estimating concordance within closed loops using $\mathrm{R}$ software. ${ }^{20}$ We further checked clinical and methodological variables to search for potential reasons that contribute to inconsistency.

\section{Additional analyses}

To enhance the clinical applicability of our report, we performed subgroup analyses to investigate the rank ordering of $H$. pylori eradication regimens among countries with high and low clarithromycin resistance. We performed a literature review to determine the prevalence of clarithromycin resistance of countries where RCTs were conducted from literature published in the last 10 years. Accordingly, all included RCTs were categorised into high or low clarithromycin resistant group using resistant rate of $15 \%$ as cut-off. The rank of each treatment was determined in both groups. Besides, we examined weighted average eradication rates for all regimens in each country. The results were categorised by clarithromycin resistance rate.

Providing that antibiotic resistant rate increases over time and may cause bias to the results, we categorised all RCTs into 2005-2010 and 2011-2016 and investigated the relative efficacies of regimens in both subgroups. Furthermore, we conducted sensitivity test by omitting studies with one or more treatment arms of $<50$ patients, high heterogeneity and reporting bias as they might substantially affect the results. ${ }^{21}$

\section{RESULTS}

We initially identified 7130 citations from the literature (figure 1). Of these, we excluded 6835 articles after the title and abstract screening. Of the remaining 295 articles, 178 were excluded due to duplication, non-RCT, unavailability of full text, ineligible study design, employment of therapy other than the 17 study $H$. pylori eradication therapies and comparison of different PPIs or bismuth. Finally, 117 studies were eligible for inclusion in the network meta-analysis.

The major characteristics of the included RCTs are listed in the online supplementary appendix 1 table S1. In total, 32852 treatment-naïve participants were recruited. The mean age of these patients was 47.75. The total number of participant in each trial ranged from 59 to 1463 . The mean sample size for each trial was 280.56 subjects. Most studies assessed H. pylori infection status at 4-12 weeks after the completion of therapy, except one trial that detects outcome at 14 weeks. We conducted internal validity assessment for each study included in the meta-analysis (see online supplementary appendix 1 figure S1). Inadequate allocation concealment was the main source of potential bias in our meta-analysis.

The network of comparison of the 17 therapies is described in figure 2. In total, 117 studies comprising 266 comparison arms were enrolled. Figure 3 summarises the pooled estimates of the results of network meta-analysis for the effectiveness of H. pylori eradication. Among all of the therapies, ST-14 $(\mathrm{OR}=3.74,95 \% \mathrm{CrI} 2.37$ to 5.96$)$ presented the highest efficacies. These were followed by $\mathrm{HY} \geq 10(\mathrm{OR}=2.94,95 \% \mathrm{CrI} 1.93$ to 4.49$)$, mBQT-14 (OR=2.87, 95\% CrI 1.61 to 5.17$), \mathrm{CT} \geq 10$ $(\mathrm{OR}=2.80,95 \% \mathrm{CrI} 2.07$ to 3.82$)$, BQT-14 $(\mathrm{OR}=2.64,95 \%$ CrI 1.21 to 5.78$), \mathrm{BQT} \leq 10(\mathrm{OR}=2.60,95 \% \mathrm{CrI} 1.75$ to 3.87$)$, TT-Q $\geq 10 \quad(\mathrm{OR}=2.31, \quad 95 \% \quad \mathrm{CrI} \quad 1.52$ to 3.53$), \quad \mathrm{CT} \leq 7$ $(\mathrm{OR}=2.08,95 \% \mathrm{CrI} 1.51$ to 2.91$)$ and $\mathrm{ST} \leq 10(\mathrm{OR}=2.04,95 \%$ CrI 1.68 to 2.49 ) all of which were at least two times more effective than TT-C-7. TT-M-7 (OR=0.82, 95\% CrI 0.56 to 1.19) represented the least efficacious regimen among all regimens.

Regimens of longer duration tended to demonstrate higher effectiveness when compared with regimens of shorter duration (figure 4). The OR for the comparison between ST-14 and $\mathrm{ST} \leq 10$ was 1.84 (95\% CrI 1.17 to 2.90$)$. Longer duration of quinolone-based triple therapy (TT-Q $\geq 10)$ was superior to shorter duration of quinolone-based triple therapy (TT-Q-7), with OR of 1.91 (95\% CrI 1.23 to 2.96). TT-C-14 (OR=1.72, 95\% CrI 1.37 to 2.17$)$ and TT-C-10 $(\mathrm{OR}=1.32,95 \% \mathrm{CrI} 1.04$ to 1.69$)$ presented higher eradication rate when compared with TT-C-7. The effectiveness of most triple therapies was inferior to alternative regimens such as quadruple therapy, ST, CT and HY.

We also categorised included studies into two groups according to the resistance rate to clarithromycin in the respective countries (see online supplementary appendix 2 table S1). Among included studies, 76 trials were classified into 'highclarithromycin resistance group' while 36 trials were categorised into 'low-clarithromycin resistant group'. In the former group, ST-14 $(\mathrm{OR}=6.53,95 \% \mathrm{CrI} 3.23$ to 13.63$)$ revealed the highest efficacies, followed by $\mathrm{BQT} \leq 10(\mathrm{OR}=3.64,95 \% \mathrm{CrI} 2.07$ to 6.48), BQT-14 (OR=3.49, 95\% CrI 1.48 to 8.30$)$ and mBQT-14 (OR=3.33, 95\% CrI 1.79 to 6.29$)$. Among countries with low clarithromycin resistance, $\mathrm{HY} \geq 10 \quad(\mathrm{OR}=2.85,95 \%$ CrI 1.58 to 5.37 ) presented highest OR, followed by $\mathrm{CT} \geq 10$ $(\mathrm{OR}=2.43,95 \%$ CrI 1.55 to 3.88$)$ and ST-14 $(\mathrm{OR}=2.24,95 \%$ CrI 1.29 to 3.93 ) (online supplementary appendix 2 table S2a, $\mathrm{S} 2 \mathrm{~b})$. We reviewed all included trials and calculated the average eradication rate weighted by the number of participants (see online supplementary appendix 1 table S2). When compared with other regimens, 7-day triple therapies achieved therapeutic rate in few countries. In addition, countries such as Turkey that 
Figure 1 Literature search and selection flow diagram.

\section{0 potentially relevant citations identified \\ 5783 PubMed}

1319 Cochrane Central Register of Controlled Trials

28 Additional relevant citations identified from review articles and manual search

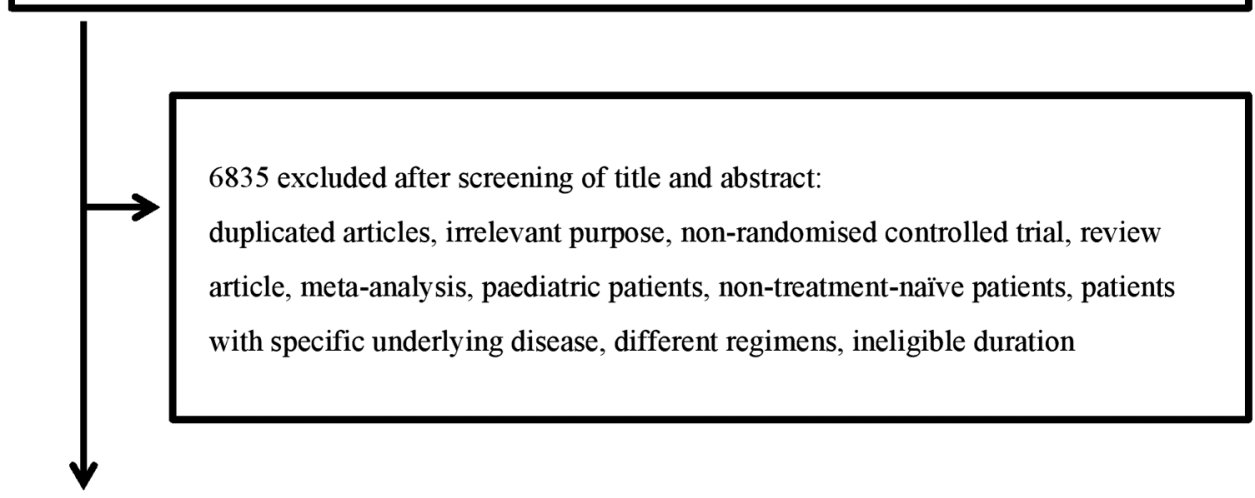

295 retrieved articles extracted for detailed assessment

178 articles excluded with reasons of

10 duplicated articles or interim results of published articles

52 abstract only or poster

18 review article, commentary, observational study, poster, or no identical title/first author found in literature searching

2 ineligible study design (e.g. non-randomised trial, placebo-based trial)

9 ineligible participants (e.g. paediatric, non-first line, gastric adenoma)

61 assessing other treatments (e.g. regimens with histamine 2 receptor antagonists, regimens without proton pump inhibitor,

furazolidone/rifabutin/azithromycin-based regimens, dual therapy, reversed hybrid therapy, therapy with probiotics or herbs)

26 comparing different proton pump inhibitors or bismuth within same regimens

117 full-text articles extracted for detailed assessment and data synthesis presented low eradication rate in most triple therapies should consider more advanced therapies as first-line treatment.

Acknowledging that resistant rate increase over time, we also performed subgroup analysis based on of publication year (see online supplementary figure S2). ST-14 was ranked the first and second place in 2005-2010 and 2011-2016 subgroups, respectively. The rank of mBQT-14 rose from sixth in 2005-2010 group to first in 2011-2016 group. However, this results needs to be interpreted in cautious, as there was only one trial that compared mBQT-14 in 2005-2010 group. Most triple therapies ranked low in both 2011-2016 category and 2005-2010 category. Sensitivity analyses demonstrated that omissions of studies with low numbers of patients in treatment arms and reporting bias only minimally affected on the rank ordering of the 17 regimens (see online supplementary table S3). However, BQT emerged as top three regimens in the effectiveness ranking when trials with high heterogeneity were excluded.

Estimations of pooled ORs for $H$. pylori eradication by traditional meta-analysis, heterogeneity analyses and publication bias are illustrated (see online supplementary figure S3). Overall, the 


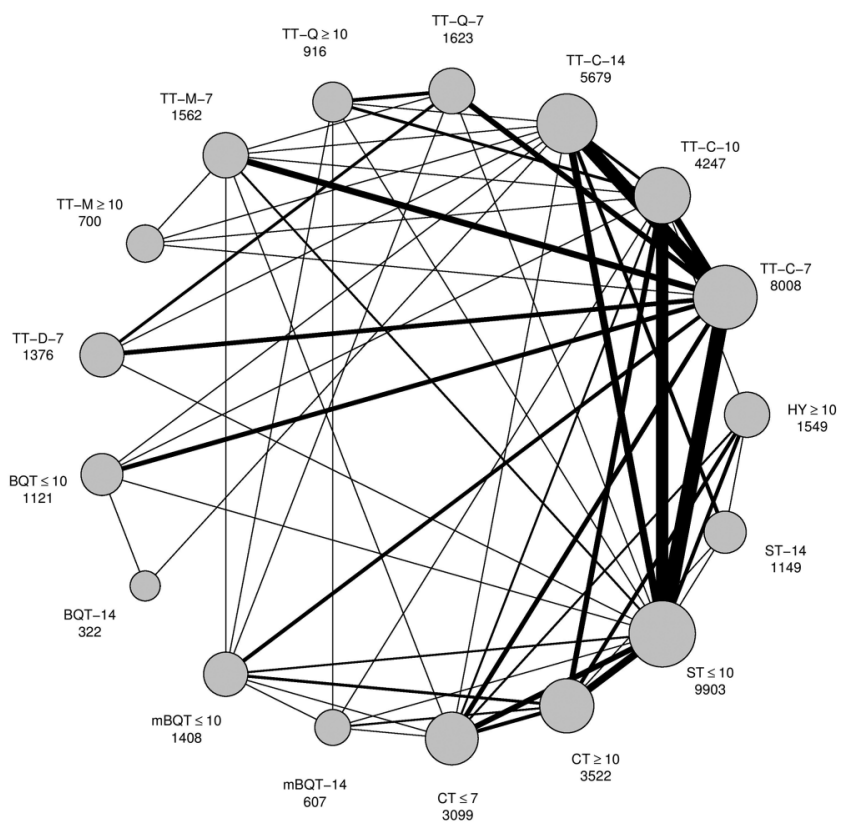

Number of Studies

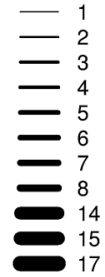

Abbreviations

TT-C-7: clarithromycin-based triple therapy for 7 days

TT-C-10: clarithromycin-based triple therapy for 10 days

TT-C-14: clarithromycin-based triple therapy for 14 days

TT-Q-7: quinolone-based triple therapy for 7 days

$T T-Q \geq 10$ : quinolone-based triple therapy for more than or equal to 10 days

TT-M-7: metronidazole-based triple therapy for 7 days

$T T-M \geq 10$ : metronidazole-based triple therapy for more than or equal to 10 days

TT-D-7: double major drugs triple therapy for 7 days

$\mathrm{BQT} \leq 10$ : bismuth-containing quadruple therapy for less than or equal to 10 days

BQT-14: bismuth-containing quadruple therapy for 14 days

$\mathrm{mBQT} \leq 10$ : modified bismuth-containing quadruple therapy for less than or equal to 10 days

mBQT-14: modified bismuth-containing quadruple therapy for 14 days

$\mathrm{CT} \leq 7$ : concomitant therapy for less than or equal to 7 days

$\mathrm{CT} \geq 10$ : concomitant therapy for more than or equal to 10 days

$\mathrm{ST} \leq 10$ : sequential therapy for less than or equal to 10 days

ST-14: sequential therapy for 14 days

$H Y \geq 10$ : hybrid therapy for more than or equal to 10 days

Figure 2 Network of comparisons for Bayesian meta-analysis. Each circle represents an included regimen. The area of each of the circles is proportional to the number of subjects who received the regimen (numbers of subjects are shown below the regimens). Each line denotes direct comparison between two regimens. The width of the connecting lines is proportional to the number of trials (1-17). The abbreviations of the 17 regimens are listed next to the figure.

Figure 3 Order of ORs for Helicobacter pylori eradicative regimens. The forest plot illustrates the rank order of all comparators according to their efficacies. $\mathrm{Crl}$ denotes credible interval. The abbreviations of the regimens are listed in figure 2.

$\begin{array}{ll}\text { Treatment arm } & \\ & \text { OR }(95 \% \text { Crl }) \\ \text { ST-14 } & 3.74(2.37-5.96) \\ \text { HY } \geq 10 & 2.94(1.93-4.49) \\ \text { mBQT-14 } & 2.87(1.61-5.17) \\ \text { CT } \geq 10 & 2.80(2.06-3.82) \\ \text { BQT-14 } & 2.64(1.21-5.78) \\ \text { BQT } \leq 10 & 2.60(1.75-3.87) \\ \text { TT-Q } \geq 10 & 2.31(1.52-3.53) \\ \text { CT } \leq 7 & 2.08(1.51-2.90) \\ \text { ST } \leq 10 & 2.04(1.68-2.49) \\ \text { mBQT } \leq 10 & 1.88(1.28-2.77) \\ \text { TT-D-7 } & 1.80(1.19-2.76) \\ \text { TT-C-14 } & 1.72(1.37-2.17) \\ \text { TT-C-10 } & 1.32(1.04-1.69) \\ \text { TT-M } \geq 10 & 1.29(0.56-3.01) \\ \text { TT-Q-7 } & 1.21(0.86-1.71) \\ \text { TT-C-7 } & \text { Reference } \\ \text { TT-M-7 } & 0.82(0.56-1.19)\end{array}$

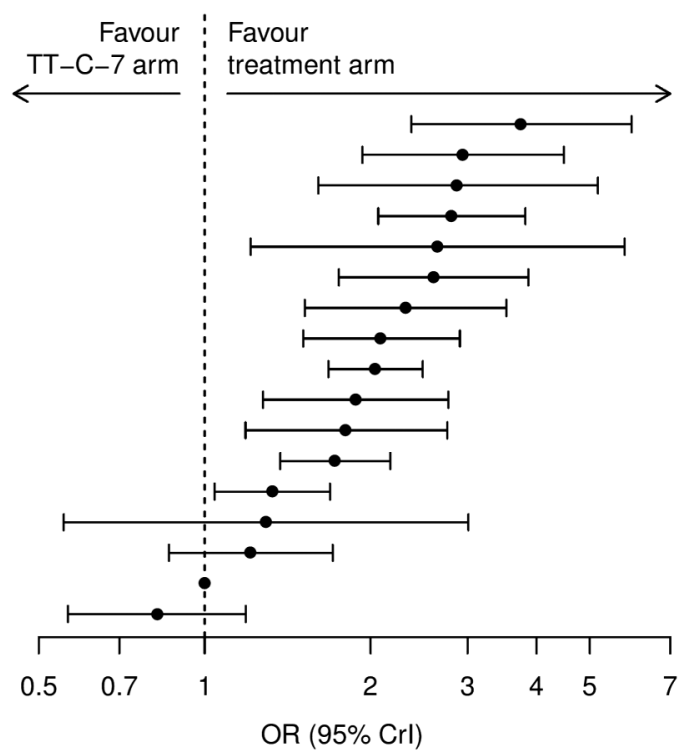

(longer or equal to 10 days) were associated with higher rate of events that lead to discontinuation.

mojority of comparisons yielded moderate to severe heterogeneity. According to inconsistency analysis, inconsistency was observed in only a few cases: 15 of the total 83 loops (see online supplementary figure S4). Of note, loops that present inconsistencies were constituted of comparisons that comprised very few trials. Therefore, the extent of inconsistency did not seem profound enough to impact the effect size of the estimates. Regarding publication bias, visual inspection of our netfunnel plot did not reveal marked asymmetry (see online supplementary figure S5).

We also reviewed severe adverse events or events that lead to withdrawal (see online supplementary table S4). Severe adverse events were rare for all regimens. Events that lead to discontinuation were observed in 57 trials. Regimens with longer duration

\section{DISCUSSION}

In the present study, we systematically compared 17 major regimens for $H$. pylori eradication to determine the optimal treatment for treatment-naïve patients. We demonstrated the highest eradication efficacies for ST-14 in countries with high clarithromycin resistance and $\mathrm{HY} \geq 10$ in countries with low clarithromycin resistance. We found that regimens of longer duration tend to have higher effectiveness than regimens of shorter duration. Besides, conventional regimens showed lower effectiveness than alternative regimens. Higher risk of events that lead to withdrawal was observed in regimens of longer duration. The 


\begin{tabular}{|c|c|c|c|c|c|c|c|c|c|c|c|c|c|c|c|c|}
\hline TT-C-7 & $\begin{array}{c}1.35 \\
(1.09-1.67) \\
\end{array}$ & $\begin{array}{c}1.74 \\
(1.26-2.41) \\
\end{array}$ & $\begin{array}{c}1.16 \\
(0.67-2.01) \\
\end{array}$ & & $\begin{array}{c}0.86 \\
(0.55-1.35) \\
\end{array}$ & $\begin{array}{c}1.60 \\
(0.89-2.87) \\
\end{array}$ & $\begin{array}{c}1.77 \\
(1.25-2.49) \\
\end{array}$ & $\begin{array}{c}1.90 \\
(0.91-3.97)\end{array}$ & & \begin{tabular}{|c|}
1.54 \\
$(0.64-3.71)$ \\
\end{tabular} & & & & $68-247$ & & \\
\hline $\begin{array}{c}1.32 \\
(1.04-1.69)\end{array}$ & $T T-C-10$ & $\begin{array}{c}1.99 \\
(1.07-3.72)\end{array}$ & & $\left(\begin{array}{c}1.92 \\
(1.29-2.87)\end{array}\right.$ & $\begin{array}{c}0.57 \\
(0.31-1.08)\end{array}$ & $\begin{array}{c}1.07 \\
(0.57-2.01)\end{array}$ & & $\begin{array}{l}3.35 \\
63-6.877)\end{array}$ & & & $\left(\begin{array}{c}0.55 \\
(0.24-1.25)\end{array}\right.$ & $1.62-5.6$ & $\begin{array}{c}1.84 \\
(1.11-3.06)\end{array}$ & $\begin{array}{c}1.35 \\
(1.00-1.81)\end{array}$ & & \\
\hline $\begin{array}{c}1.72 \\
(1.37-2.17) \\
\end{array}$ & $\begin{array}{c}1.30 \\
(0.97-1.74)\end{array}$ & TT-C-14 & & $\begin{array}{c}0.66 \\
(0.24-1.86)\end{array}$ & $\begin{array}{c}0.10 \\
(0.02-0.44)\end{array}$ & $\begin{array}{c}0.19 \\
(0.04-0.83)\end{array}$ & $\begin{array}{c}1.27 \\
(0.69-2.33)\end{array}$ & $\begin{array}{c}1.83 \\
(1.07-3.11) \\
\end{array}$ & $\begin{array}{c}1.72 \\
(1.01-2.94)\end{array}$ & & & $\begin{array}{c}0.61 \\
(0.45-0.82) \\
\end{array}$ & & \begin{tabular}{|c|}
1.16 \\
$(0.87-1.56)$ \\
\end{tabular} & & \\
\hline $\begin{array}{c}1.21 \\
(0.86-1.71) \\
\end{array}$ & $\begin{array}{c}0.91 \\
(0.62-1.35) \\
\end{array}$ & $\begin{array}{c}0.70 \\
(0.47-1.04) \\
\end{array}$ & TT-Q-7 & $\begin{array}{c}2.13 \\
1.16-3.91\end{array}$ & $\begin{array}{c}0.50 \\
(0.20-1.24) \\
\end{array}$ & & $\begin{array}{c}1.31 \\
(0.63-2.73) \\
\end{array}$ & & & $\left.\begin{array}{c}0.86 \\
(0.36-2.03)\end{array}\right)$ & & & & \begin{tabular}{c|}
1.16 \\
$(0.67-2.01)$
\end{tabular} & & \\
\hline $\begin{array}{c}231 \\
1.52-3.53\end{array}$ & $\begin{array}{c}1.75 \\
(1.14-2.67) \\
\end{array}$ & $\begin{array}{c}1.34 \\
(0.85-2.10)\end{array}$ & $\begin{array}{c}1.91 \\
(1.23-2.96) \\
\end{array}$ & $\pi-Q \geq 10$ & & & & & & $\begin{array}{c}3.40 \\
(1.03-11.22)\end{array}$ & \begin{tabular}{|c|}
1.46 \\
$(0.61-3.52)$ \\
\end{tabular} & & & $\begin{array}{c}0.29 \\
(0.18-0.49)\end{array}$ & & \\
\hline $\begin{array}{c}0.82 \\
(0.56-1.19) \\
\end{array}$ & $\begin{array}{c}0.62 \\
(0.41-0.94) \\
\end{array}$ & $\begin{array}{c}0.48 \\
(0.31-0.72) \\
\end{array}$ & $\begin{array}{c}0.68 \\
(0.42-1.10) \\
\end{array}$ & $\begin{array}{c}0.35 \\
(0.21-0.61)\end{array}$ & $T-M-7$ & $\begin{array}{c}1.87 \\
(1.05-3.30) \\
\end{array}$ & & & & \begin{tabular}{c|}
1.72 \\
$(0.77-3.83)$ \\
\end{tabular} & & $\begin{array}{c}1.75 \\
(1.07-2.86) \\
\end{array}$ & & \begin{tabular}{c|}
2.59 \\
$(0.41-16.33$ \\
\end{tabular} & & \\
\hline $\begin{array}{c}1.29 \\
(0.56-3.01) \\
\end{array}$ & $\begin{array}{c}0.97 \\
(0.41-2.30) \\
\end{array}$ & \begin{tabular}{|c|}
0.75 \\
$(0.32-1.77)$ \\
\end{tabular} & $\begin{array}{c}1.07 \\
(0.43-2.63) \\
\end{array}$ & \begin{tabular}{c|}
0.56 \\
$(0.22-1.42)$ \\
\end{tabular} & $\begin{array}{c}1.58 \\
(0.66-3.79)\end{array}$ & $T T-M \geq 10$ & & & & & & & & & & \\
\hline $\begin{array}{c}1.80 \\
(1.19-2.76) \\
\end{array}$ & $\begin{array}{c}1.36 \\
(0.85-2.19)\end{array}$ & $\left(\begin{array}{c}1.05 \\
0.66-1.66)\end{array}\right.$ & $\begin{array}{c}1.49 \\
(0.92-2.43)\end{array}$ & $\begin{array}{c}0.78 \\
(0.44-1.38) \\
\end{array}$ & $\begin{array}{l}2.20 \\
27-3.84 \\
\end{array}$ & $\begin{array}{c}1.40 \\
(0.55-3.58) \\
\end{array}$ & TT-D-7 & & & & & & & \begin{tabular}{|c|}
0.83 \\
$(0.45-1.53)$ \\
\end{tabular} & & \\
\hline & $\begin{array}{c}1.97 \\
\text { (1.27-3.03) } \\
\end{array}$ & $\begin{array}{c}1.51 \\
(0.99-2.31) \\
\end{array}$ & $\begin{array}{c}2.15 \\
1.28-3.54\end{array}$ & $\begin{array}{c}1.12 \\
(0.64-1.98) \\
\end{array}$ & $\begin{array}{c}3.18 \\
86-5.4 \\
\end{array}$ & $\begin{array}{c}2.02 \\
(0.80-5.09) \\
\end{array}$ & $\begin{array}{c}1.45 \\
(0.81-2.55) \\
\end{array}$ & BQT $\leq 10$ & $\begin{array}{c}0.87 \\
(0.43-1.78) \\
\end{array}$ & & & & & $\begin{array}{c}0.66 \\
(0.32-1.39) \\
\end{array}$ & & \\
\hline $\begin{array}{l}264 \\
21-5.78\end{array}$ & $\begin{array}{c}2.00 \\
(0.90-4.47) \\
\end{array}$ & $\begin{array}{c}1.54 \\
(0.71-3.31) \\
\end{array}$ & $\begin{array}{c}2.18 \\
(0.94-5.11)\end{array}$ & $\begin{array}{c}1.14 \\
(0.48-2.74) \\
\end{array}$ & $\begin{array}{c}3.23 \\
37-7.6 \\
\end{array}$ & $\begin{array}{c}2.05 \\
(0.65-6.43)\end{array}$ & $\begin{array}{c}1.47 \\
(0.61-3.53) \\
\end{array}$ & $\begin{array}{c}1.01 \\
(0.47-2.23) \\
\end{array}$ & BQT-14 & & & & & & & \\
\hline $\begin{array}{c}1.88 \\
(1.28-2.77) \\
\end{array}$ & $\begin{array}{c}1.42 \\
(0.94-2.15)\end{array}$ & $\begin{array}{c}1.09 \\
(0.71-1.67) \\
\end{array}$ & $\begin{array}{c}1.55 \\
(0.96-2.52)\end{array}$ & $\begin{array}{c}0.81 \\
(0.48-1.37) \\
\end{array}$ & $\begin{array}{c}2.29 \\
38-3.8 \\
\end{array}$ & $\begin{array}{c}1.45 \\
(0.58-3.64) \\
\end{array}$ & $\begin{array}{c}1.04 \\
(0.59-1.82) \\
\end{array}$ & $\begin{array}{c}0.72 \\
(0.42-1.25) \\
\end{array}$ & $\begin{array}{c}0.71 \\
(0.30-1.68) \\
\end{array}$ & $\mathrm{mBQT} \leq 10$ & & $\begin{array}{c}1.13 \\
(0.50-2.56) \\
\end{array}$ & $\begin{array}{c}1.33 \\
(0.68-2.58) \\
\end{array}$ & $\begin{array}{c}1.14 \\
(0.64-2.03) \\
\end{array}$ & & \\
\hline 61 & $\begin{array}{c}2.16 \\
(1.22-3.89 \\
\end{array}$ & $\begin{array}{c}1.66 \\
(0.91-3.06) \\
\end{array}$ & $\begin{array}{r}2.37 \\
1.24-4.5 \\
\end{array}$ & $\begin{array}{c}1.24 \\
(0.66-2.37) \\
\end{array}$ & $\begin{array}{c}3.50 \\
79-6.9\end{array}$ & $\begin{array}{c}2.22 \\
(0.81-6.15) \\
\end{array}$ & $\begin{array}{c}1.59 \\
(0.78-3.23) \\
\end{array}$ & $\begin{array}{c}1.10 \\
(0.55-2.22)\end{array}$ & $\begin{array}{c}1.09 \\
(0.41-2.84)\end{array}$ & $\left(\begin{array}{c}1.53 \\
(0.80-2.92)\end{array}\right.$ & mBQT-14 & $\begin{array}{c}0.38 \\
(0.14-1.06)\end{array}$ & $\begin{array}{c}0.84 \\
(0.47-1.50) \\
\end{array}$ & $\begin{array}{c}0.77 \\
(0.38-1.56)\end{array}$ & & \\
\hline $\begin{array}{l}2.08 \\
51-2.9\end{array}$ & $\begin{array}{c}1.57 \\
(1.11-2.25) \\
\end{array}$ & $\begin{array}{c}1.21 \\
(0.84-1.75)\end{array}$ & $\begin{array}{c}1.72 \\
\text { (1.10-2.73) }\end{array}$ & $\begin{array}{c}0.90 \\
(0.55-1.50)\end{array}$ & $\begin{array}{l}2.54 \\
1.60-4.0\end{array}$ & $\begin{array}{c}1.61 \\
(0.66-3.97)\end{array}$ & $\begin{array}{c}1.16 \\
(0.69-1.96)\end{array}$ & $\begin{array}{c}0.80 \\
(0.49-1.32) \\
\end{array}$ & $\begin{array}{c}0.79 \\
(0.34-1.82) \\
\end{array}$ & $\begin{array}{c}1.11 \\
(0.70-1.78)\end{array}$ & $\begin{array}{c}0.73 \\
(0.39-1.35) \\
\end{array}$ & $\mathrm{CT} \leq 7$ & $\begin{array}{c}1.78 \\
(1.03-3.05)\end{array}$ & $\begin{array}{c}1.10 \\
(0.69-1.76)\end{array}$ & & $\begin{array}{c}0.98 \\
(0.50-1.91) \\
\end{array}$ \\
\hline $06-3.8$ & $\begin{array}{r}211 \\
(1.57-2.86)\end{array}$ & $\begin{array}{c}1.63 \\
(1.16-2.28)\end{array}$ & $\begin{array}{c}2.31 \\
1.50-3.57 \\
\end{array}$ & $\left(\begin{array}{c}1.21 \\
(0.76-1.95)\end{array}\right.$ & $\begin{array}{r}3.42 \\
.17-5.4 \\
\end{array}$ & $\begin{array}{c}2.17 \\
(0.90-5.27) \\
\end{array}$ & $\begin{array}{c}1.55 \\
(0.94-2.58)\end{array}$ & $\begin{array}{c}1.07 \\
(0.67-1.75)\end{array}$ & $\begin{array}{c}1.06 \\
(0.47-2.42)\end{array}$ & $\begin{array}{r}1.49 \\
(0.98-2.27)\end{array}$ & $\begin{array}{c}0.98 \\
(0.55-1.74)\end{array}$ & $\left(\begin{array}{c}1.34 \\
(0.92-1.95)\end{array}\right.$ & $\mathrm{CT} \geq 10$ & $\left(\begin{array}{c}0.83 \\
(0.60-1.14)\end{array}\right.$ & \begin{tabular}{c|c}
0.74 \\
$(0.35-1.56)$
\end{tabular} & $\begin{array}{c}0.94 \\
(0.68-1.28) \\
\end{array}$ \\
\hline & $\begin{array}{c}1.54 \\
(1.23-1.93)\end{array}$ & $\mid \begin{array}{c}1.18 \\
(0.93-1.51)\end{array}$ & $\begin{array}{c}1.68 \\
(1.17-2.43)\end{array}$ & $\begin{array}{c}0.88 \\
(0.58-1.34) \\
\end{array}$ & $\begin{array}{c}2.49 \\
1.67-3.7 \\
\end{array}$ & $\begin{array}{c}1.58 \\
(0.67-3.72)\end{array}$ & $\begin{array}{c}1.13 \\
(0.72-1.77)\end{array}$ & $\begin{array}{c}0.78 \\
(0.52-1.19) \\
\end{array}$ & $\begin{array}{c}0.77 \\
(0.35-1.70)\end{array}$ & $\left(\begin{array}{c}1.08 \\
(0.73-1.61)\end{array}\right.$ & $\begin{array}{c}0.71 \\
(0.40-1.25)\end{array}$ & $\begin{array}{c}0.98 \\
(0.71-1.34)\end{array}$ & $\begin{array}{c}0.73 \\
(0.55-0.96)\end{array}$ & $\mathrm{ST} \leq 10$ & $\left(\begin{array}{c}1.45 \\
(0.87-2.43)\end{array}\right.$ & $\left(\begin{array}{c}1.17 \\
(0.41-3.32)\end{array}\right.$ \\
\hline $\begin{array}{l}3.74 \\
37-5.96\end{array}$ & $\begin{array}{c}2.83 \\
(1.75-4.59)\end{array}$ & $\begin{array}{r}2.17 \\
(1.42-3.35 \\
\end{array}$ & $\begin{array}{r}3.09 \\
(1.78-5.42 \\
\end{array}$ & $\begin{array}{c}1.61 \\
(0.89-2.96)\end{array}$ & $\begin{array}{l}4.57 \\
.58-8.17 \\
\end{array}$ & $\begin{array}{r}2.90 \\
\text { (1. 112-7.51 } \\
\end{array}$ & $\begin{array}{r}2.08 \\
(1.13-3.83 \\
\end{array}$ & $\begin{array}{c}1.44 \\
(0.81-2.59) \\
\end{array}$ & $\begin{array}{c}1.42 \\
(0.59-3.42)\end{array}$ & $\begin{array}{c}2.00 \\
(1.13-3.54)\end{array}$ & $\begin{array}{c}1.30 \\
(0.64-2.65)\end{array}$ & $\begin{array}{c}1.80 \\
(1.06-3.05)\end{array}$ & $\begin{array}{c}1.34 \\
(0.82-2.18) \\
\end{array}$ & $\begin{array}{c}1.84 \\
(1.17-2.90) \\
\end{array}$ & ST -14 & $\begin{array}{c}1.09 \\
(0.52-2.26)\end{array}$ \\
\hline $\begin{array}{l}2.94 \\
93-4.45\end{array}$ & $\begin{array}{c}2.22 \\
(1.45-3.40 \\
\end{array}$ & $\begin{array}{c}1.71 \\
(1.10-2.65)\end{array}$ & $\begin{array}{r}2.43 \\
1.44-4.1\end{array}$ & $\begin{array}{c}1.27 \\
(0.73-2.23)\end{array}$ & $\begin{array}{r}3.59 \\
2.09-6.1\end{array}$ & $\begin{array}{c}2.28 \\
(0.90-5.79)\end{array}$ & $\begin{array}{c}1.63 \\
(0.91-2.92)\end{array}$ & $\begin{array}{c}1.13 \\
(0.65-1.99)\end{array}$ & $\begin{array}{c}1.11 \\
(0.46-2.66)\end{array}$ & $\mid \begin{array}{c}1.56 \\
0.92-2.66)\end{array}$ & $\begin{array}{c}1.02 \\
(0.53-2.00)\end{array}$ & $\left(\begin{array}{c}1.41 \\
(0.89-2.24)\end{array}\right.$ & $\begin{array}{c}1.05 \\
(0.70-1.57)\end{array}$ & $\left|\begin{array}{c}1.44 \\
(0.97-2.14)\end{array}\right|$ & \begin{tabular}{|c|c}
0.78 \\
$(0.46-1.35)$
\end{tabular} & $H Y \geq 10$ \\
\hline
\end{tabular}

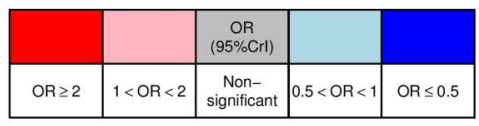

Figure 4 Comparative efficacies of Helicobacter pylori eradication regimens by league table. Left lower: the row-defining regimens are compared with the column-defining regimens by network meta-analyses. OR of more than one reflects increased effectiveness by the row-defining regimens. For example, the cell at the intersection of column 1 and row 3 indicates that the eradication rate of clarithromycin-based triple therapy for 14 days (TT-C-14) is significantly higher than that of clarithromycin-based triple therapy for 7 days (TT-C-7) with $0 \mathrm{R}=1.72$ (95\% Crl 1.37 to 2.17). Right upper: the column-defining regimens are compared with the row-defining regimens by traditional meta-analyses. Cells with red and pink background denote ORs significantly higher than 2.00 and range between 1.01 and 1.99, respectively. Cells with dark blue and light blue backgrounds denote ORs significantly lower than 0.50 and range between 0.99 and 0.51 , respectively. Cells with grey backgrounds denote ORs with non-significant difference. The abbreviations of the regimens are listed in figure 2 . Crl, credible interval.

therapeutic eradication rate according to clarithromycin resistance rate also provided clinically meaningful information for decision-making. Taken together, our study presents comprehensive comparisons of $H$. pylori eradication therapies according to clarithromycin resistance rate and provides significant insights into the compositional effects of regimen type and duration, adverse events and therapeutic cure rate.

Using Bayesian network meta-analysis, which integrates the advantages of direct and indirect evidence, we were able to obtain more accurate estimations. In contrast to traditional meta-analysis, network meta-analysis allows for the simultaneous assessment of more than two types of interventions. Furthermore, it provides estimates of comparative effect among all regimens, including those that have not been compared head-to-head in the literature. Moreover, the large pooled patient size contributed substantial statistical advantage by enhancing the power of our study, as well as to the robustness of our analyses. As the geometry provides a nice graphical overview of the proportion of participants in different regimens and where the gaps were, the authors were well placed to propose research priorities. According to a recent consensus, inclusion of earlier literature, when antibiotic resistance rate was low, could introduce bias. ${ }^{13}$ Therefore, we only included articles from 2005 onwards.

To our knowledge, this is the most comprehensive network meta-analysis of RCTs in assessing optimal therapy for H. pylori eradication. In previous network meta-analyses, therapies were grouped into three comparators ${ }^{22}$ or 14 comparators. $^{12}$ Although the latter comprehensively compared estimated effects of different regimens, the authors included different kinds of probiotics-containing and $\mathrm{H} 2$ blockers-containing regimens in the network. Moreover, they grouped clarithromycin-based therapy with metronidazole-based therapy, which might not provide crucial clinical information to clinicians. The results that the effectiveness of probiotics-supplemented TT was significantly greater than 14 days HY and 10 or 14 days ST were not comparable to clinical observations.

The results of the present study that longer duration increases eradication rate are consistent with the findings of previous analyses. Yuan et al reported that prolonged duration of PPI-based TT significantly increases eradication rate. ${ }^{24}$ In the present guidelines, it is suggested that the duration of clarithromycin-based TT be extended as previous meta-analyses have indicated that 10-day and 14-day treatment regimens improve $H$. pylori eradication rate when compared with 7-day treatment regimen. ${ }^{4} 25-27$ According to World Gastroenterology Organisation global guidelines for lowincome and middle-income countries, however, an optimal duration for $H$. pylori eradication remains controversial. Taking into consideration local clarithromycin resistance, cost-effectiveness and compliance, some research groups have suggested 7-day treatment while some have favoured 10-day therapy. ${ }^{1}$ 
The superiority of newly developed regimens, such as ST, CT and HY, has been confirmed in the present study. ST was developed in Italy in $2000 .{ }^{28}$ Previous meta-analyses reported that ST demonstrated higher effectiveness than TT. ${ }^{29} 30$ Since most clinical trials of ST enrolled in our meta-analyses have been conducted in Italy, Asia-Pacific consensus guidelines in 2009 suggested that more evidence is needed to support ST as an alternative to clarithromycin-based TT in Asian countries. ${ }^{31}$ Recently, three meta-analyses from Asia comparing the efficacies of ST and standard TT have shown significant but modest increase in eradication rate following $\mathrm{ST}^{32-34}$ In a recent meta-analysis, 10-day ST is superior to 7-day TT (relative risk (RR) $1.21,95 \%$ CI 1.17 to 1.25 ), marginally superior to 10 -day TT (RR 1.11, 95\% CI 1.04 to 1.19) and inferior to 14-day TT (RR 1.00 , 95\% CI 0.94 to 1.06 ). ${ }^{35}$ Our results showed that both ST-14 and ST $\leq 10$ are superior to all 7 -day, 10-day and 14-day TT, with the exception that TT-Q $\geq 10$ demonstrated higher effectiveness than $\mathrm{ST} \leq 10$. Among countries with high clarithromycin resistance, the OR of ST-14 rose substantially to 6.53 (95\% CrI 3.23 to 13.63), while the rank of ST-14 dropped to third place among countries with low clarithromycin resistance. The use of ST as first-line therapy is in line with two guidelines, ${ }^{46}$ but challenges the other one. ${ }^{13}$ Therefore, the use of optimal regimen requires other considerations such as local antibiotic resistance and cost. Besides, it is 10-day but not 14-day ST being recommended in current guidelines. Since there were only five trials that compared ST-14, all of which were from Asian countries, more trials are needed in order to yield a confirmed conclusion.

CT is less complex than ST and HY with the administration of four drugs at the same time. ${ }^{37} 38$ From a meta-analysis of six clinical trials, most of which were published before 2001, the effectiveness of CT is higher than that of standard therapy (OR $2.40,95 \%$ CI 1.63 to 3.55$).{ }^{39}$ On the contrary, Greenberg et al published one large trial in seven Latin American sites and concluded that 5-day CT is inferior to 14-day clarithromycin-based TT. ${ }^{40}$ Therefore, it has been suggested that elevated antibiotic resistance impedes the success of 5-day CT and that longer treatment duration is warranted. ${ }^{39}$ The results of the present network meta-analysis support this hypothesis, as we find that $\mathrm{CT} \geq 10$ is more effective than all TT. The eradication effect of $\mathrm{CT} \geq 10$ was ranked as the fifth and second choice in countries with high and low clarithromycin resistance, respectively.

HY was first proposed in 2011, and its effectiveness has been compared with ST and CT. ${ }^{41}$ Two meta-analyses have suggested similar eradication rates for these newer treatments. ${ }^{42} 43$ Our results suggested that $H Y \geq 10$ has comparable effectiveness to mBQT-14 and $C T \geq 10$. The estimated effects of $H Y \geq 10$ rose significantly to become the optimal regimen when to compare with TT-C-7 in the low clarithromycin resistant group. However, the results should be interpreted cautiously as there were only three trials that studied $H Y \geq 10$ in this subgroup.

The effectiveness of BQT and quinolone-based TT are also examined in the present study. Quinolone-based TT and BQT are effective as a second-line or third-line rescue therapy, as recommended in Maastricht Guidelines IV and the Toronto Consensus. ${ }^{4} 13$ Our findings supported the guidelines that TT-Q $\geq 10$ and BQTs were superior to clarithromycin-based TT. In a recent meta-analysis that compared TT and BQT, Venerito et al indicated a non-significant difference between TT and BQT given in 7-day or 10-day to 14 -day regimens. ${ }^{44}$ This observation conflicted with our findings, as we showed that CT $\geq 10$ was superior to TT-C-14 and that CT $\geq 7$ was superior to TT-C-7. However, this could be explained by the publication year of included studies. Studies that were published after 2005 in previous meta-analysis supported that $\mathrm{CT} \geq 10$ had higher effectiveness than TT-C $\geq 10 .^{45} 46$ In addition, we showed that BQTs presented high relative efficacies in high clarithromycin resistance group. This is consistent to previous studies that clarithromycin resistance does not influence the effectiveness of BQT. $^{44} 47$

A key assumption of network meta-analysis is the transitivity between trials. ${ }^{48}$ The baseline characteristics and potential effect modifiers seemed broadly similar across regimens. However, we were not able to explore the distribution of smokers because many studies that did not report on this. Although certain extent of clinical heterogeneity is unavoidable, we have tried to minimise the effect by using strict inclusion criteria and excluding articles that were published before 2005 .

There are several limitations to the present study. First, we only investigated mainstream eradication regimens in treatment-naïve patients. Non-mainstream regimens (eg, furazolidone-based regimen) were thus not included in this study. Second, inadequate concealment and blinding may elicit bias and impact the size of the estimate. Subgroup analysis to detect the effect of inadequate concealment was not performed because less than half (44 studies) of the included studies reported adequate concealment. Third, the overall heterogeneity in this network meta-analysis was moderate to severe. Excluding studies with high heterogeneity influenced the ranking of regimens, which might due to fewer studies in some pairwise comparisons. Fourth, although inconsistency is low in our study, there is no sufficient evidence to prove that this is the conclusive evidence of network consistency. This is owing to the random effect model used in our study and the moderate level of between-study heterogeneity. Finally, synthesis of evidence under the condition of multiple comparisons may mitigate homogeneity and thereby affect further interpretation. We enrolled RCTs from the recent 10 years to avoid potential bias posed by increasing resistance rate with the aid of washout period and outcome measurement to enhance the homogeneity of included trials.

In conclusion, ST and HY appeared to be the most effective therapies in countries with high and low clarithromycin resistance, respectively. Our network meta-analysis provides practical implications that may be translated into clinical benefit. The rank order, when interpreted concerning different local antibiotics resistant rates, tolerability and local therapeutic rate, may help physicians in determining favourable first-line therapy regimen and duration for $H$. pylori eradication.

\section{Author affiliations}

${ }^{1}$ Division of Gastroenterology and Hepatology, Taichung Veterans General Hospital, Taichung, Taiwan

${ }^{2}$ Nuffield Department of Population Health, University of Oxford, Oxford, UK

${ }^{3}$ Blatvatnik School of Government, University of Oxford, Oxford, UK

${ }^{4} S$ chool of Medicine, Fu Jen Catholic University, New Taipei City, Taiwan

${ }^{5}$ Center for Health Policy Research and Development, Miaoli, Taiwan

${ }^{6}$ National Cancer Institute, National Health Research Institutes, Miaoli, Taiwan

${ }^{7}$ Department of Internal Medicine, National Taiwan University Hospital, Taipei, Taiwan

${ }^{8}$ Faculty of Medicine and Institute of Clinical Medicine, National Yang-Ming University, Taipei, Taiwan

${ }^{9}$ Department of Public Health and Graduate Institute of Clinical Medical Science, China Medical University, Taichung, Taiwan

${ }^{10}$ Department of Life Sciences and Rong Hsing Research Center for Translational Medicine, National Chung-Hsing University, Taichung, Taiwan

Acknowledgements We would like to thank all of the members of the Taiwan Gastrointestinal Disease and Helicobacter Consortium (the member list is provided in the online supplementary appendix) for their valuable discussion of this manuscript. We would also like to thank Professor Yu-Kang, Tu from National Taiwan University and Dr Anna Chaimani from University of loannina for their comments and suggestions. 
Collaborators Consortium members are listed in the online supplementary appendix.

Contributors C-YW, YHY, J-TL and M-SW designed the meta-analysis, with the inputs of all listed authors. YHY, S-IS and BZ contributed to data acquisition and drafted the article. YHY, S-IS and HJH contributed to data analysis and interpretation. All authors performed critical revision of the manuscript and approved the final draft of the article.

Funding This work was supported in part by the National Health Research Institutes (PH-104-PP-23), Ministry of Science and Technology, Executive Yuan, ROC, Taiwan (MOST-103-2314-B-030-004-MY2) and Taichung Veterans General Hospital (TCVGH-1043303C), Taiwan.

Competing interests None declared.

Ethics approval The institutional review boards of Taichung Veterans General Hospital.

Provenance and peer review Not commissioned; externally peer reviewed.

\section{REFERENCES}

1 Hunt RH, Xiao SD, Megraud F, et al. World Gastroenterology Organization. Helicobacter pylori in developing countries. World Gastroenterology Organisation Global Guideline. J Gastrointestin Liver Dis 2011:20:299-304.

$2 \mathrm{McColl} \mathrm{KE}$. Clinical practice. Helicobacter pylori infection. N Engl J Med 2010;362:1597-604.

3 de Martel C, Ferlay J, Franceschi S, et al. Global burden of cancers attributable to infections in 2008: a review and synthetic analysis. Lancet Oncol 2012;13:607-15.

4 Malfertheiner P, Megraud F, O'Morain CA, et al. Management of Helicobacter pylori infection-the Maastricht IV/ Florence Consensus Report. Gut 2012:61:646-64.

5 De Francesco V, Giorgio F, Hassan C, et al. Worldwide H. pylori antibiotic resistance: a systematic review. J Gastrointestin Liver Dis 2010:19:409-14.

6 Megraud F, Coenen S, Versporten A, et al. Helicobacter pylori resistance to antibiotics in Europe and its relationship to antibiotic consumption. Gut 2013;62:34-42.

7 Sasaki H, Nagahara A, Hojo M, et al. Ten-year trend of the cumulative Helicobacter pylori eradication rate for the 'Japanese eradication strategy'. Digestion 2013;88:272-8.

8 Howden CW, Hunt RH. Guidelines for the management of Helicobacter pylori infection. Ad Hoc Committee on Practice Parameters of the American College of Gastroenterology. Am J Gastroenterol 1998:93:2330-8.

9 Ades AE, Sculpher $M$, Sutton $A$, et al. Bayesian methods for evidence synthesis in cost-effectiveness analysis. PharmacoEconomics 2006;24:1-19.

10 Song F, Altman DG, Glenny AM, et al. Validity of indirect comparison for estimating efficacy of competing interventions: empirical evidence from published meta-analyses. BMJ 2003;326:472.

11 Sutton $A$, Ades $A E$, Cooper $N$, et al. Use of indirect and mixed treatment comparisons for technology assessment. PharmacoEconomics 2008;26:753-67.

12 Li BZ, Threapleton DE, Wang JY, et al. Comparative effectiveness and tolerance of treatments for Helicobacter pylori: systematic review and network meta-analysis. BMJ 2015;351:h4052.

13 Fallone CA, Chiba N, van Zanten SV, et al. The Toronto Consensus for the Treatment of Helicobacter pylori Infection in Adults. Gastroenterology 2016;151:51-69.e14.

14 Higgins JPT, Altman D, Sterne JAC. Assessing risk of bias in included studies. In: Higgins JPT, Green S, eds. Cochrane handbook for systematic reviews of interventions: Cochrane book series. Chichester: John Wiley \& Sons, 2008:187-241.

15 Hutton B, Salanti G, Caldwell DM, et al. The PRISMA extension statement for reporting of systematic reviews incorporating network meta-analyses of health care interventions: checklist and explanations. Ann Intern Med 2015;162:777-84.

16 DerSimonian R, Laird N. Meta-analysis in clinical trials. Control Clin Trials 1986:7:177-88.

17 Higgins JP, Thompson SG, Deeks JJ, et al. Measuring inconsistency in meta-analyses. BMJ 2003:327:557-60.

18 Chaimani A, Higgins JP, Mavridis D, et al. Graphical tools for network meta-analysis in STATA. PLOS ONE 2013;8:e76654.

19 Caldwell DM, Gibb DM, Ades AE. Validity of indirect comparisons in meta-analysis. Lancet 2007;369:270; author reply 1.

20 Salanti G, Marinho V, Higgins JP. A case study of multiple-treatments meta-analysis demonstrates that covariates should be considered. J Clin Epidemiol 2009;62:857-64.

21 Chan AW, Hróbjartsson A, Haahr MT, et al. Empirical evidence for selective reporting of outcomes in randomized trials: comparison of protocols to published articles. JAMA 2004;291:2457-65.

22 Rodriguez EA, Donath $\mathrm{E}$, Sussman DA. Eradication and adherence rates among helicobacter pylori regimens—a network meta-analysis. Gastroenterology 2014;146: S-557
23 McNicholl AG NO, Gisbert JP. Sequential and concomitant treatments in H. pylori eradication: a network meta-analysis. Gastroenterology 2014;146:S-395.

24 Yuan Y, Ford AC, Khan KJ, et al. Optimum duration of regimens for Helicobacter pylori eradication. Cochrane Database Syst Rev 2013;(12):CD008337.

25 Calvet X, García N, López T, et al. A meta-analysis of short versus long therapy with a proton pump inhibitor, clarithromycin and either metronidazole or amoxycillin for treating Helicobacter pylori infection. Aliment Pharmacol Ther 2000;14:603-9.

26 Ford A, Moayyedi P. How can the current strategies for Helicobacter pylori eradication therapy be improved? Can J Gastroenterol 2003:17(Suppl B):36B-40B.

27 Fuccio L, Minardi ME, Zagari RM, et al. Meta-analysis: duration of first-line proton-pump inhibitor based triple therapy for Helicobacter pylori eradication. Ann Intern Med 2007:147:553-62.

28 Zullo A, Rinaldi V, Winn S, et al. A new highly effective short-term therapy schedule for Helicobacter pylori eradication. Aliment Pharmacol Ther 2000;14:715-18.

29 Jafri NS, Hornung CA, Howden CW. Meta-analysis: sequential therapy appears superior to standard therapy for Helicobacter pylori infection in patients naive to treatment. Ann Intern Med 2008;148:923-31.

30 Zullo A, De Francesco V, Hassan C, et al. The sequential therapy regimen for Helicobacter pylori eradication: a pooled-data analysis. Gut 2007;56: 1353-7.

31 Fock KM, Katelaris P, Sugano K, et al. Second Asia-Pacific Consensus Guidelines for Helicobacter pylori infection. J Gastroenterol Hepatol 2009:24:1587-600.

32 Chung JW, Ha M, Yun SC, et al. Meta-analysis: sequential therapy is superior to conventional therapy for Helicobacter pylori infection in Korea. Korean J Gastroenterol 2013;62:267-71.

$33 \mathrm{Kim} J \mathrm{~S}, \mathrm{Kim}$ BW, Ham JH, et al. Sequential therapy for helicobacter pylori infection in Korea: systematic review and meta-analysis. Gut Liver 2013;7:546-51.

34 Yoon $\mathrm{H}$, Lee $\mathrm{DH}$, Kim N, et al. Meta-analysis: is sequential therapy superior to standard triple therapy for Helicobacter pylori infection in Asian adults? J Gastroenterol Hepatol 2013;28:1801-9.

35 Gatta L, Vakil N, Vaira D, et al. Global eradication rates for Helicobacter pylori infection: systematic review and meta-analysis of sequential therapy. BMJ 2013;347: f4587.

36 Chey WD, Wong BC, Practice Parameters Committee of the American College of Gastroenterology. American College of Gastroenterology guideline on the management of Helicobacter pylori infection. Am J Gastroenterol 2007; 102:1808-25

37 Okada M, Oki K, Shirotani T, et al. A new quadruple therapy for the eradication of Helicobacter pylori. Effect of pretreatment with omeprazole on the cure rate. I Gastroenterol 1998;33:640-5.

38 Treiber G, Ammon S, Schneider E, et al. Amoxicillin/metronidazole/omeprazole/ clarithromycin: a new, short quadruple therapy for Helicobacter pylori eradication. Helicobacter 1998;3:54-8.

39 Gisbert JP, Calvet X. Update on non-bismuth quadruple (concomitant) therapy for eradication of Helicobacter pylori. Clin Exp Gastroenterol 2012;5:23-34.

40 Greenberg ER, Anderson GL, Morgan DR, et al. 14-day triple, 5-day concomitant and 10-day sequential therapies for Helicobacter pylori infection in seven Latin American sites: a randomised trial. Lancet 2011:378:507-14.

41 Hsu PI, Wu DC, Wu JY, et al. Modified sequential Helicobacter pylori therapy: proton pump inhibitor and amoxicillin for 14 days with clarithromycin and metronidazole added as a quadruple (hybrid) therapy for the final 7 days. Helicobacter 2011;16:139-45.

42 He L, Deng T, Luo H. Meta-analysis of sequential, concomitant and hybrid therapy for Helicobacter pylori eradication. Intern Med 2015;54:703-10.

43 Wang B, Wang YH, Lv ZF, et al. Review: efficacy and safety of hybrid therapy for Helicobacter pylori infection: a systematic review and meta-analysis. Helicobacter 2015;20:79-88

44 Venerito $M$, Krieger T, Ecker $T$, et al. Meta-analysis of bismuth quadruple therapy versus clarithromycin triple therapy for empiric primary treatment of Helicobacter pylori infection. Digestion 2013;88:33-45.

45 Songür $Y$, Senol A, Balkarli A, et al. Triple or quadruple tetracycline-based therapies versus standard triple treatment for Helicobacter pylori treatment. Am J Med Sci 2009;338:50-3

46 Uygun A, Kadayifci A, Safali M, et al. The efficacy of bismuth containing quadruple therapy as a first-line treatment option for Helicobacter pylori. J Dig Dis 2007:8:211-5.

47 Katelaris PH, Forbes GM, Talley NJ, et al. A randomized comparison of quadruple and triple therapies for Helicobacter pylori eradication: the QUADRATE Study. Gastroenterology 2002;123:1763-9.

48 Salanti G. Indirect and mixed-treatment comparison, network, or multiple-treatments meta-analysis: many names, many benefits, many concerns for the next generation evidence synthesis tool. Res Synth Methods 2012;3:80-97. 